\title{
Evolution of Energy Storage on the Platform of Supercapacitors Katsuhiko NAOI*
}

\author{
Department of Applied Chemistry, Tokyo University of Agriculture \& Technology, \\ 2-24-16 Naka-cho, Koganei, Tokyo 184-8558, Japan \\ *Corresponding author: k-naoi@cc.tuat.ac.jp
}

\section{ABSTRACT}

There has been a major effort, all over the world, to increase an energy density of electrochemical capacitors to meet more demands for electric automotive and regenerative energy storage applications. Hybridizing batterycapacitor electrodes can certainly overcome the energy density limitation of the conventional electrochemical capacitors because they employ both the system of a battery-like (redox) and a capacitor-like (double-layer) electrode, producing a larger working voltage and capacitance. Nanoscience and nanotechnology can provide tremendous benefits to electrochemical energy storage devices, such as batteries and supercapacitors, by combining new nanoscale properties to realize enhanced energy and power capabilities. There are increasing number of published reports on hybrid systems. Several potential strategies to enhance the energy density above that of Gen. 1 supercapacitors is now being discussed and some fundamental issues and future directions for Gen. 2 $\& 3$ will be identified in near future.

(c) The Electrochemical Society of Japan, All rights reserved.

Keywords : Hybrid Supercapacitor, Nano-technology, Smart Mobility, Wireless Society

\section{Overview}

Dynamic energy storage by supercapacitors is an important energy and environmental technology that is highly influential in advancing our future automotive and wireless society. ${ }^{1-3}$ Unlike batteries, supercapacitors are efficient energy storage devices that exhibit high power capability, long cycle life, long calendar life, and wide operational temperature ranges. ${ }^{4-10}$ Figure 1 compares many different characteristics of Li-ion battery and supercapacitor, showing that, except for the energy density, supercapacitors are superior in most of the items than Li-ion batteries. Thus, the supercapacitor is regarded as a reliable, durable and safe technology ${ }^{1-6}$ with increasing effectiveness when utilized in smart mobility and wireless applications. ${ }^{6,11}$

However, since the energy density of the current supercapacitors lies below $10 \mathrm{Wh} \mathrm{L}^{-1}$, their uses are limited and cannot fully meet performance demands required by existing electronic appliances and electronic devices. In the past decade, supercapacitors have been vigorously researched worldwide in hopes to improve their energy density. In this special issue, there are about 30 contributions dealing with new electrode materials, new cell configurations, new electrolyte systems and other innovations relative to the recent advances in supercapacitors' technology.

Among them, hybrid approaches combining lithium-ion battery (LIB) and supercapacitor (EDLC) electrodes can overcome the energy density limitation ${ }^{6,10}$ and can double or triple the energy density of the conventional electrochemical capacitors (see Fig. 2).

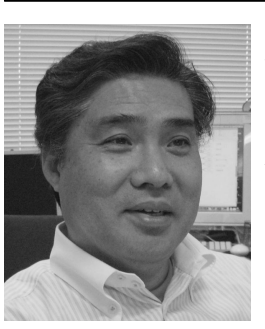

Katsuhiko Naoi is a professor of chemistry at Tokyo University of Agriculture \& Technology (TAT). With a Ph.D. from Waseda University, Tokyo, his research interests are advanced supercapacitors, future nanobatteries, for automotive/stationary applications and energy, environmental, and materials science. He serves as vice chair of The Capacitor Technology Committee of The Electrochemical Society of Japan.

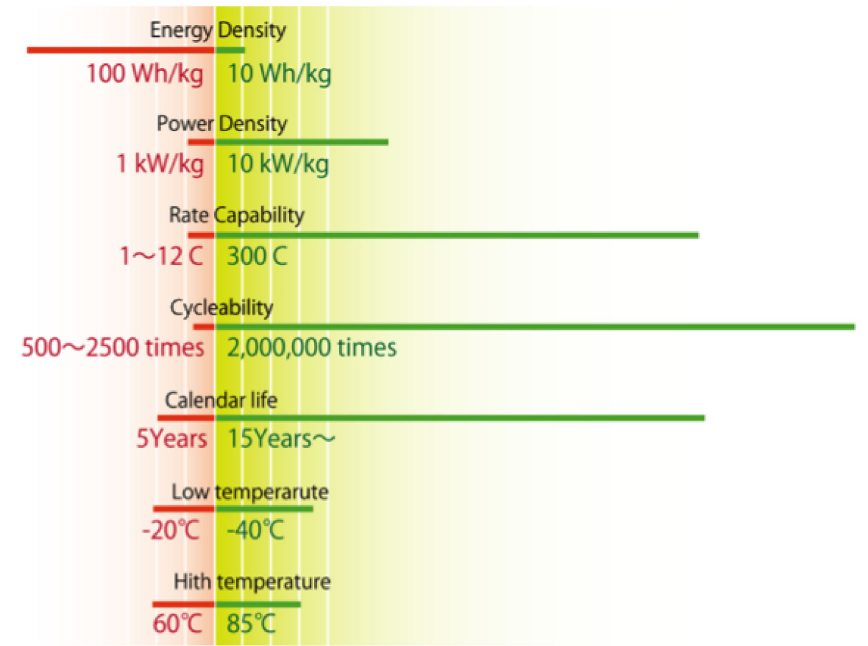

Figure 1. (Color online) Li-ion battery (red lines) vs. Supercapacitor (green lines) comparison of their features.

However, the charge-discharge rates at the faradic electrodes must be increased to the levels of non-faradic electrodes in order to balance the two different systems. To meet these demands, stateof-the-art nano-carbon materials are actively applied to form composites enhancing the energy-power capability effectively. ${ }^{12,13}$ As shown in Fig. 3, the Gen.1 supercapacitors utilize activated carbons for both their positive and negative electrodes that show non-faradaic, double-layer charging-discharging mechanism in symmetric configuration. With this system, the Gen.1 supercapacitor is extremely efficient regarding rapid charge-discharge characteristics compared with batteries. Hybridizing battery and capacitor electrodes has already started as Gen.2 hybrids (LIC: lithium-ion capacitor and NHC: NanoHybrid capacitor ${ }^{14-19}$ ). Ultrafast battery materials overcome the energy density limitation of existing Gen.1 capacitors without much sacrificing the cycling performances. Normal battery-capacitor hybrids employ high-energy \& sluggish 

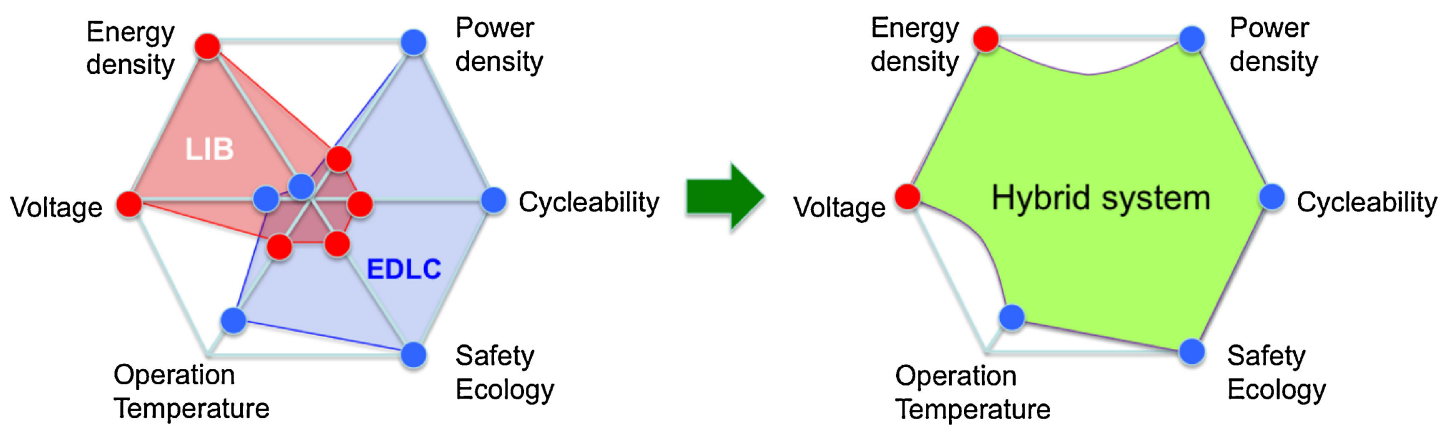

Figure 2. (Color online) A big demand for higher energy density for new applications necessitate the hybridization of batteries, specifically, LIB and EDLCs into hybrid devices having both the attributes of lithium ion batteries, allowing high voltage and energy density, and the characteristics of electrochemical capacitors of high power, longer cycleability, wider temperature operation and safety issues.
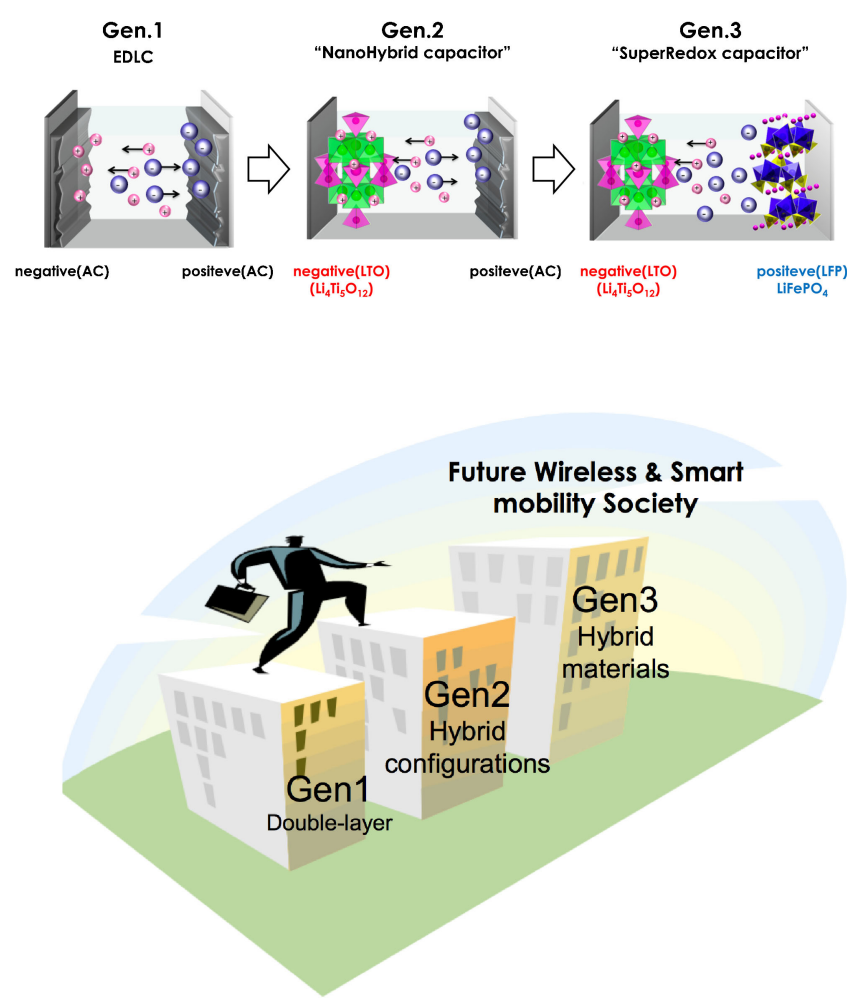

Figure 3. (Color online) Evolution of supercapacitors from Gen.1 (double-layer capacitors) to Gen.2 (half \& half), and to Gen.3 (fused battery-capacitor materials). The concept of hybrids, i.e., the configuration in the electrode configuration and the materials can overcome the energy-power density limitations over existing Gen.1 supercapacitors.

redox electrode and low-energy \& fast double-layer electrodes, possibly producing a larger working voltage and higher over-all capacitance. In order to smoothly operate such asymmetric systems, however, the rates of the two different electrodes must be highly balanced. Especially, the redox rates of the battery electrodes must be substantially increased to the levels of double-layer process. We attempt to identify the essential issues for the realizable hybrids and suggest ways to overcome the rate enhancement by exemplifying ultrafast performance of the $\mathrm{Li}_{4} \mathrm{Ti}_{5} \mathrm{O}_{12}$ nanocrystal prepared via a unique in-situ material processing technology under ultra-centrifuging.

\section{Outlook}

Nanoscience and nanotechnology can provide tremendous benefits to electrochemical energy storage devices, such as batteries and supercapacitors, by combining new nanoscale properties to realize enhanced energy and power capabilities. There are increasing number of published reports on hybrid systems. Several potential strategies to enhance the energy density above that of Gen.1 supercapacitors is now being discussed and some fundamental issues and future directions will be identified in near future.

\section{References}

1. P. Simon and Y. Gogotsi, Nat. Mater., 7, 845 (2008).

2. J. Chmiola, C. Largeot, P.-L. Taberna, P. Simon, and Y. Gogotsi, Science, 328, 480 (2010).

3. W. Gao, N. Singh, L. Song, Z. Liu, A. L. M. Reddy, L. Ci, R. Vajtai, Q. Zhang, B. Wei, and P. M. Ajayan, Nat. Nanotechnol., 6, 496 (2011).

4. D. Pech, M. Brunet, H. Durou, P. Huang, V. Mochalin, Y. Gogotsi, P.-L. Taberna, and P. Simon, Nat. Nanotechnol., 5, 651 (2010).

5. S. W. Lee, N. Yabuuchi, B. M. Gallant, S. Chen, B.-S. Kim, P. T. Hammond, and Y. Shao-Horn, Nat. Nanotechnol., 5, 531 (2010).

6. A. Burke, J. Power Sources, 91, 37 (2000).

7. A. G. Pandolfo and A. F. Hollenkamp, J. Power Sources, 157, 11 (2006).

8. R. Kötz and M. Carlen, Electrochim. Acta, 45, 2483 (2000).

9. I. Plitz, A. Dupasquier, F. Badway, J. Gural, N. Pereira, A. Gmitter, and G. G. Amatucci, Appl. Phys. A, 82, 615 (2006).

10. K. Naoi and P. Simon, Interface, 17, 34 (2008).

11. X. Lang, A. Hirata, T. Fujita, and M. Chen, Nat. Nanotechnol., 6, 232 (2011).

12. Q. Wang, Z. Wen, and J. Li, J. Nanosci. Nanotechnol., 7, 3328 (2007).

13. W.-D. Zhang, B. Xu, and L. C. Jiang, J. Mater. Chem., 20, 6383 (2010).

14. K. Naoi, S. Ishimoto, N. Ogihara, Y. Nakagawa, and S. Hatta, J. Electrochem. Soc., 156, A52 (2009).

15. K. Naoi, S. Ishimoto, Y. Isobe, and S. Aoyagi, J. Power Sources, 195, 6250 (2010).

16. K. Naoi, Fuel Cells, 10, 825 (2010).

17. K. Naoi, S. Ishimoto, J. Miyamoto, and W. Naoi, Energy Environ. Sci., 5, 9363 (2012).

18. K. Naoi, W. Naoi, S. Aoyagi, J. Miyamoto, and T. Kamino, Acc. Chem. Res., 46, 1075 (2013)

19. K. Naoi, Y. Nagano, and W. Naoi, TANSO, 256, 22 (2013). 